\title{
Application of Computer Simulation in the Prediction of Fatigue Properties of Rubber Materials
}

\author{
Xiao Jing \\ School of Mechanical and Material Engineering, Jiujiang University,Jiujiang,332005,China
}

shuimuxintaizi@163.com

Keywords: Rubber, Fatigue life, Finite Element.

\begin{abstract}
Rubber products are widely used, and some of them have no obvious plastic deformation on surface, there exists huge risks, so the prediction of the fatigue life of rubber materials is very important. In this paper, the fatigue life of rubber materials is accurately analyzed by using advanced computer technology. It not only analyzes the fatigue life curve (S-N curve), but also measures the change of the stress distribution with ABAQUS finite element analysis software. The results show that, this method is accurate and effective and can provide scientific and accurate data for the prediction of rubber fatigue.
\end{abstract}

\section{Introduction}

Rubber fatigue is one of the important parameters in measuring the physical and mechanical properties of the rubber[1]. Under the action of the external force or the periodic deformation, periodic load will appear, which may result in brittle failure. Although there is no apparent plastic deformation on surface, there exists obvious danger. Aiming at the problem, this paper makes full use of the computer related technology to carry out further study. Firstly, it carries out the mechanical property of the rubber. In this way, it will acquire the data of the volume compression, uniaxial tension, plane shear and other related data. With the combination of the scientific constitutive model and the acquired data, this paper fits out the parameters of the constitutive model. The paper also obtains the fatigue life curve (S-N Curve) with the constitutive acceleration test method[2]. The principle of the process is the elastic behavior superposition. This paper uses ABAQUS finite element software to analyze the result of the stress change and distribution. It also analyzes and calculates the result with the FE-SAFE fatigue software. The analytic result can be imported to the ABAQUS software through ODB and finally achieve the rubber fatigue failure data[3].

\section{Principle of the Rubber Fatigue Prediction}

For the rubber materials with the same property [4], if I1, I2, I3 are the three basic invariants for the Cauchy deformation tensor Green, and there is no stress topography super-elastic material, the strain energy function $\mathrm{W}$ will be as follows:

$$
\begin{aligned}
W & =W\left(I_{1}, I_{2}, I_{3}\right) \\
I_{1} & =\operatorname{tr} C=C_{i j}=\lambda_{1}^{2}+\lambda_{2}^{2}+\lambda_{3}^{2} \\
I_{2} & =\frac{1}{2}\left[(\operatorname{tr} C)^{2}-\operatorname{tr} C^{2}\right]=\frac{1}{2}\left(I_{1}^{2}-C_{i j} C_{i j}\right) \\
& =\left(\lambda_{1} \lambda_{2}\right)^{2}+\left(\lambda_{2} \lambda_{3}\right)^{2}+\left(\lambda_{3} \lambda_{1}\right)^{2} \quad i, j=1,2,3 \\
I_{3} & =\operatorname{det} C=\left(\lambda_{1} \lambda_{2} \lambda_{3}\right)^{2} \\
C & =F^{T} F
\end{aligned}
$$




$$
\begin{aligned}
& F=\frac{\partial x}{\partial X} \\
& J=\operatorname{det} F
\end{aligned}
$$

In the function, I represents the second order tensor invariant, $\mathrm{J}$ is the volume ratio of the rubber material before and after deformation, $\mathrm{F}$ represents the inherent deformation gradient, $\mathrm{x}$, and $\mathrm{X}$ are the coordinates of the same point before and after modification of a sample, the lower 1,2,3 correspond to axis $\mathrm{X}, \mathrm{Y}$ and $\mathrm{Z}$ [5]

$\mathrm{N}$ order polynomial theory is the so-called constitutive model derived from the phenomenological theory. If the property of the materials is the same, the strain energy density parameters can be broken into two parameters which are the volumetric strain energy and the strain deviator energy. It can be expressed as follows:

$$
W=f\left(\bar{I}_{1}-3, \bar{I}_{2}-3\right)+g(J-1)
$$

If $g=\sum_{i=1}^{N} \frac{1}{D_{i}}(J-1)^{2 i}$,the strain energy can be obtained with Taylor formula.

$$
W=\sum_{i+j=1}^{N} C_{i j}(\bar{I}-3)^{i}(\bar{I}-3)^{j}+\sum_{i=1}^{N} \frac{1}{D}(j-1)^{2 i}
$$

Equation (9) is the rubber constitutive model, in which $\mathrm{N}$ is the order number of the polynomial. Suppose $\mathrm{k}_{0}$ is the initial volume modulus, and $\mu_{0}$ is the shear modulus, then there is the following equation.

$$
\mu_{0}=2\left(C_{10}+C_{01}\right), k=2 / D_{1}
$$

The strain energy function is obtained through a series of experiments by Scholar Mooney with the use of material phase transition theory. It can be expressed as:

$$
W=C_{10}\left(I_{1}-3\right)+C_{01}\left(I_{2}-3\right)
$$

The Rivlin quadratic equation can be expressed as

$$
W=C_{10}\left(I_{1}-3\right)+C_{01}\left(I_{2}-3\right)+C_{11}\left(I_{1}-3\right)+C_{20}\left(I_{1}-3\right)^{2}+C_{02}\left(I_{2}-3\right)^{2}
$$

Suppose the rubber material is the moderate strain, there will be an inflection point in the stress strain curve in equation (12), then the fit will be perfect. Ogden model can be obtained through the phenomenological theory[7] ,abd the constitutive model with the main elongation as the main variables can be expressed as:

$$
W=\sum_{i=1}^{n} \frac{2 \mu_{i}}{a_{i}^{2}}\left(\bar{\lambda}_{1}^{a_{i}}+\bar{\lambda}_{2}^{a_{i}}+\bar{\lambda}_{3}^{a_{i}}-3\right)+\sum_{i=1}^{n} \frac{1}{D}(J-1)^{2 i}
$$

In equation (13), $\bar{\lambda}_{i}=J^{-\frac{1}{3}} \lambda_{i} \rightarrow \bar{\lambda}_{1} \bar{\lambda}_{2} \bar{\lambda}_{3}=1$ can accurately describe the strain stress and strain state with fewer items under different strain conditions. This is the main advantage of Ogden equation.

\section{Material Preparation and Mechanical Property Testing}

The rubber formulation used in the test is shown in Table 1.

Table 1 Rubber formulation

\begin{tabular}{|r|r|c|c|c|c|}
\hline NR & S & Zn0 & Stearic acid & CZ & N330 \\
\hline 100 & 2 & 4 & 2 & 1 & 50 \\
\hline
\end{tabular}

The rubber is molded into dumbbell through a certain process before testing. The uniaxial tensile stress-strain curves can be obtained through the universal testing machine[9], which can be shown in Figure 1. 


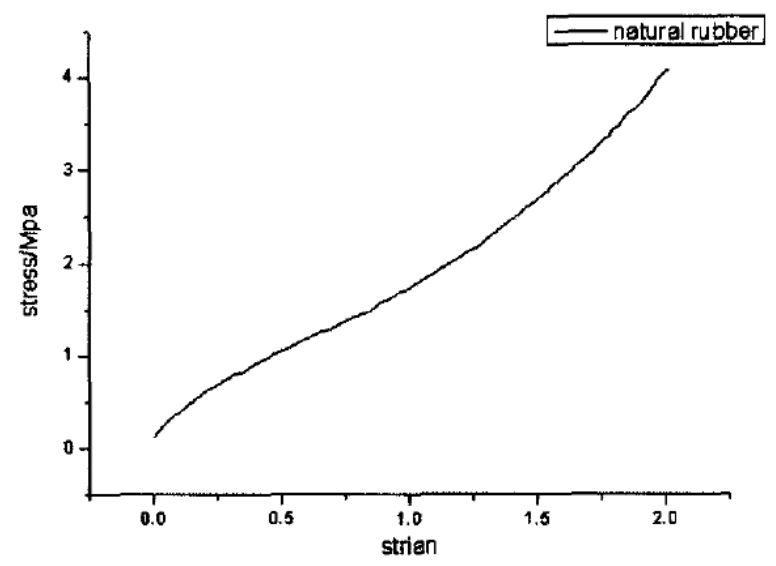

Figure 1 Stress-strain curve of the natural rubber

\section{Fatigue Test}

The specific parameters of the rubber model tested are: width $\times$ thickness $=25 \mathrm{~mm} \times 1 \mathrm{~mm}$, and the length is a rectangle changing with the target deformation region L0. During the test, the $38 \mathrm{~mm}$ position at the both ends clamped by the device do not participate in the tensile deformation, and the both near $5 \mathrm{~mm}$ range are the eliminating errors. If the model is stressed from L0 to L, the corresponding data can be obtained by using the automatic winding machine to test fatigue, as shown in table 2.

Table 2 Fatigue test data

\begin{tabular}{|c|c|c|c|c|c|}
\hline $\begin{array}{c}\text { Numbe } \\
r\end{array}$ & LO/mm & L/mm & strain & $\begin{array}{c}\text { stress/MP } \\
\text { a }\end{array}$ & $2 \mathrm{Nf}$ \\
\hline $1 \#$ & 7 & 15.97 & 1.255 & 2.16 & $\begin{array}{c}1060 \\
1\end{array}$ \\
\hline $2 \#$ & 20 & 41.87 & 1.093 & 1.88 & 10866 \\
\hline $3 \#$ & 28 & 52.92 & 0.890 & 1.57 & 22279 \\
\hline $4 \#$ & 32 & 57.07 & 0.780 & 1.40 & 27549 \\
\hline $5 \#$ & 30 & 47.73 & 0.591 & 1.17 & 41804 \\
\hline
\end{tabular}

The S-N curve can be acquired by integrating the tested data, which can provide scientific and accurate data for the FE-SAFE fatigue calculation.

\section{Modeling Analysis and Calculation}

The model is constructed with computer modeling software ABAQUS. It mainly consists of geometric modeling, given boundary conditions and rubber property by applying load, meshing and establishing analysis steps[10]. The Evaluate key of the Property in ABAQU is used to fit. The data used in the test are: Polynomial $\mathrm{N}=\mathrm{I}$ (Mooney Rivlin), Polynomial $\mathrm{N}=2$, Ogden $\mathrm{N}=3$, and the final fitting results can be shown in Figure 2 and Figure 3.

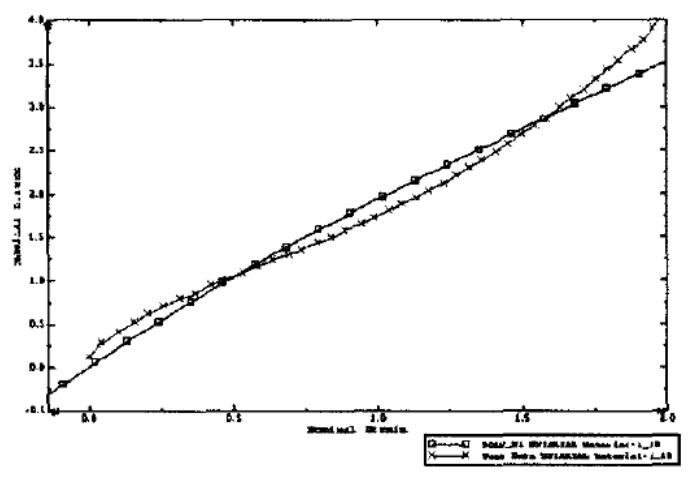

Figure 2 Polynomial N=1(Mooney Rivlin) 


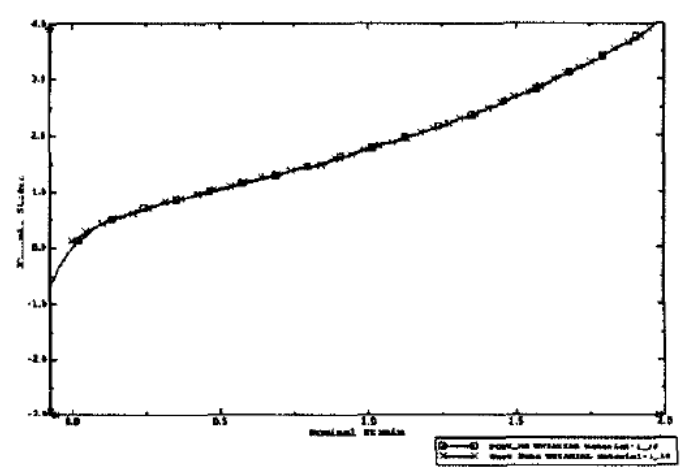

Figure 3 Polynomial $\mathrm{N}=2$

The comparison of Figure 2 and 3 shows that Polynomial $\mathrm{N}=2$ fits the data best. The finally obtained material parameters are $\mathrm{C} 10=-0.21773519, \mathrm{C} 01=3.21511525, \quad \mathrm{C} 20=0.150582960$, $\mathrm{C} 11=-0.624109235$.

\section{FE-SAFE Fatigue Result}

In using FE-SAFE software to calculate, the parameters to be set are material property, surface roughness and applied load[11]. Before calculating, SN curve must be imported to FE-SAFE software and stress fatigue algorithm will be used at the same time. Two important parameters, stress and strain correction, should be fully considered. The fatigue limit should be defined to 104 times and the surface roughness parameter should be set to $0.6 \sim 1.6 \mathrm{~m}$, the load parameter $2,-2$ which means that the boundary displacement is $40 \mathrm{~mm}$. Through the software, the fatigue life can be obtained that is 10641 times, FOS intensity factor is 1.5 , the number of fatigue life is 4.027 , which means that the times of the fracture are 10641. The data shows that the error tested is very small, and the position of the damage is fully accurate, and this test method is accurate and reliable.

\section{Conclusion}

The paper uses the basic mechanics principle and ABAQUS finite element analysis software to analyze the stress change and distribution with computer technology. It not only discusses the method of how to obtain fatigue life curve (S-N curve), but also carries out the scientific and accurate calculation by FE-SAFE fatigue calculation software. The result shows that the method is accurate and feasible and can provide scientific and accurate data for the prediction of the rubber material fatigue.

\section{Acknowledgement}

This work is supported by Science Foundation of Jiangxi Provincial Department of Education (GJJ11243), National Science Foundation of Jiangxi Province (2011BBE50011).

\section{References}

[1] Liu Jianxun, Huang Youjian \& Liu Baibing. A Rubber Elastic Element Fatigue Life Prediction Method of Research [J]. Software Engineering,2011, 34(3):12-15.

[2] Pang Hao, Liu Bing, Huang Yuhui etc.. Poly-1, 2- propylene Carbonate / Natural Rubber Blend Elastomer Formulation and Design [J]. Polymer Materials Science and Engineering, 2002,18 (2): 71-73.

[3] Hao Tong-hui, Jiang Tao, Qiu Li. Dynamically Vulcanized EPDM / PP TPV Recipe Design [J]. Elastomers, 2005,15 (2): 33-36.

[4] Zou Mingqing, Cai Dayang, LEE Wingchi etc.. Uniform Design in Tire Formulation Design [J]. Tire Industry, 1997,17 (6): 332-338. 
[5] Shi Xinglee. Development and Application of Artificial Neural Networks [J]. Chongqing University of Science and Technology, 2006,8 (2): 99 -101,110.

[6] Zhu Daqi. Artificial Neural Network Status and Prospect [J]. Jiangnan University, 2004, 3 (1), 103-110.

[7] Tang Suli, Luo Yufeng. Development and Application of Artificial Neural Network Technology [J]. Computer Development \& Application, 2009,22 (10): 59-61.

[8] Sui Ying, Fu chunju, Yan Xingyan. Development and Application of Artificial Neural Network [J]. Popular Science, 2010 (5): 20-34.

[9]Guo D, Wang Y L, Nan C W , et a1. Application of Artificial Neural Network Technique to the Formulation Design of Dielectric Ceramics[J]. Sensors and Actuators. A: Physica1. 2002, 102(1-2): 93-98.

[10]Hecht-Nielsen R. Theory of the Back Propagation NeuralNetwork[J]. Proceedings of IJCNN, 1989: 593-603.

[11] Chen Erfan, Deng Wenwen, Han Yunfeng etc.. EPDM and Other Heat Aging Behavior and BP Neural network [J]. New Chemical Materials, 2010,38 (10): 80-82,97. 